\title{
Estudio Sobre Condiciones de Dolor, Incomodidad y Enfermedad Debido a la Exposición al Frío Artificial y Controlado en Frigoríficos en el Brasil
}

\author{
STUDY OF PAIN CONDITIONS, DISCOMFORT AND DISEASE DUE TO COLD EXPOSURE TO ARTIFICIAL COLD \\ AND CONTROLLED IN SLAUGHTERHOUSES IN BRAZIL
}

\author{
Fabiano Takeda', Natália Fonseca Dias², Antônio Renato Pereira Moro³ ${ }^{3}$ Sirlene Aparecida Takeda Bresciani ${ }^{4}$ Armando \\ Monterrosa Quintero ${ }^{5}$ \\ 1. Estudiante de Posgrado en Ingeniería de Producción. Centro Tecnológico CTC, Universidad Federal de Santa Catarina (Brasil). \\ 2. Estudiante de Posgrado en Ingeniería de Producción. Centro Tecnológico CTC, Universidad Federal de Santa Catarina (Brasil). \\ 3. Profesor Programa Ingeniería de Producción. Centro Tecnológico CTC, Universidad Federal de Santa Catarina (Brasil). \\ 4. Profesora Programa Ingeniería de Producción. Centro Tecnológico, Campus Juara. Universidad del Estado de Mato Grosso. \\ 5. Estudiante de Posgrado en Biodinámica del Desempeño Humano, Centro Deportivo, CDS, Universidad Federal de Santa Catarina (Brasil).
}

\begin{abstract}
RESUMEN
Fundamentos: El objetivo de esta investigación fue analizar las quejas de dolores y la temperatura superficial de la piel en tres sitios corporales localizados en las muñecas y manos (bilateralmente) de trabajadores expuestos a los ambientes artificialmente climatizados para verificar la relación de las quejas de dolor con la temperatura. Métodos: Para la realización del estudio fueron recolectados datos cualitativos con el uso del cuestionario y datos cuantitativos con equipos de monitoreo ambiental y una cámara de detección de radiación infrarroja. El estudio fue realizado con diecinueve trabajadores de un sector de un matadero de pollos.

Resultados: Los resultados apuntan que fueron identificadas menores temperaturas superficiales de la piel, alcanzando un valor de $16,67{ }^{\circ} \mathrm{C}$ y fueron evidentes en los dedos de la mano que aseguran el producto, estando de acuerdo con el modelo adaptado de la ISO 11079 tratándose de una condición causadora de dolor.

Conclusiones: Podemos concluir que este modelo de negocio, en las circunstancias que se presentan, exponen a los trabajadores a condiciones que pueden causar malestar, dolor, accidentes y enfermedades profesionales relacionadas con la exposición al frío. Este resultado es preocupante debido a su potencial impacto en una mayor cobertura de la salud del trabajador, teniendo en cuenta que las estadísticas oficiales se refieren al crecimiento de este tipo de actividad debido al crecimiento del sector.
\end{abstract}

(Takeda F, Fonseca N, Pereira A, Takeda S, Monterrosa A, 2017. Estudio Sobre Condiciones de Dolor, Incomodidad y Enfermedad Debido a la Exposición al Frío Artificial y Controlado en Frigoríficos en el Brasil. Cienc Trab. Ene-Abr; 19 [58]: 14-19).

Palabras claves: TERMOGRAFÍA, INDUSTRIA DE PROCESAMIENTO DE ALIMENTOS, SALUD LABORAL, FRÍO.

\section{ABSTRACT}

Background: The objective of this research was to analyse the complaints about pains and the surface temperature of the skin in three body sites located in the wrists and hands (bilaterally) of workers exposed to artificially conditioned environment to verify the relationship of pain complaints with the temperature.

Methods: To conduct the study qualitative data were collected using a questionnaire and quantitative data using environmental monitoring equipment and an infrared radiation detecting camera. The study was carried out with nineteen workers in a chicken slaughterhouse sector. Results: The results indicate that the lower surface temperatures of the skin reached $16,67{ }^{\circ} \mathrm{C}$, were highlighted on the fingers that hold the product, considered according to the adapted model of ISO 11079 as a condition causing pain.

Conclusion: Therefore, it can be concluded that this business model, under the presented circumstances, expose workers to conditions that may cause discomfort, pain, accidents and occupational diseases related to exposure to cold. This result is worrying due to its potential impact on the worker's health and greater coverage, considering that that official statistics refer to the growth of this type of activity due to the sector's expansion.

Key words: THERMOGRAPHY, FOOD-PROCESSING INDUSTRY, OCCUPATIONAL HEALTH, COLD.
Correspondencia / Correspondence:

Armando Monterrosa Quintero

Universidad Federal de Santa Catarina -UFSC, Centro Deportivo- CDS,

Campus Universitario, Trindade.

Caja Postal 476, CEP 88040-900, Florianópolis, Santa Catarina, Brasil. Tel.: 05548 9833-6029

e-mail: adomonterrosa@hotmail.com (A. Monterrosa)

Recibido: 15 de Agosto de 2016 / Aceptado: 12 de diciembre de 2016

\section{INTRODUCCIÓN}

La industria Brasilera de sacrificio y procesamiento de pollos presenta un constante crecimiento en las últimas décadas, ubicándose como el tercer mayor productor mundial, destacándose la exportación de este alimento para cerca de 150 países, siendo responsable por el 1,8\% del Producto Interno Bruto (PIB) nacional. La expectativa es que, hasta el 2020, la producción nacional avícola alcance los valores de $48,1 \%$ de las exportaciones 
mundiales. ${ }^{1,2}$ Esos datos representan los sobresalientes números de empresas frigorificas y plantas de sacrificio en actividad en el territorio brasilero. En función de este escenario, la necesidad de mano de obra para atender la demanda productiva es elevada. Según la Unión Brasilera de Avicultura, la UBABEF, la avicultura emplea aproximadamente 3,6 millones de personas directa e indirectamente incorporando trabajadores del campo y de plantas frigoríficas. ${ }^{3}$ En contraposición al crecimiento avícola, notamos negativamente una grave problemática en relación a la salud de los trabajadores que laboran en los frigoríficos, evidenciada por medio de estudios nacionales e internacionales que relatan la diversidad de riesgos físicos (ruido, temperaturas extremas, humedad, vibración), químicos, biológicos y ergonómicos presentes en estas actividades. ${ }^{4-18}$

Las actuales estimativas nacionales de la protección social reportan un elevado indice, cuya medida alcanza 23\% del trabajo en el sector frigorífico, en la condición de incapacidad laboral o en la espera de decisiones judiciales debido a accidentes y enfermedades ocupacionales. ${ }^{19}$

En general, los frigoríficos el ambiente de trabajo es húmedo con presencia de ruidos provocados por gran cantidad de máquinas y exposición frecuente a bajas temperaturas. ${ }^{20}$ Para agravar, las actividades son de forma estática, lo que disminuye la generación de calor del cuerpo, debido al proceso productivo secuencial comparado con una línea de montaje, en la cual la velocidad de trabajo no es determinada por el individuo. ${ }^{21-23}$

Teniendo en cuenta el Decreto 210 de 10/11/1998 del Ministerio de Agricultura y de Suministros del Brasil, que define las actividades de cortes de carnes estableciendo límites para los sitios laborales, determinando que la temperatura del producto no puede ser superior a 7 ${ }^{\circ} \mathrm{C}$, y la temperatura ambiental no puede ser superior a los $12{ }^{\circ} \mathrm{C} .{ }^{24}$ Las temperaturas bajas en el ambiente laboral posibilitan desarrollar problemas de salud determinando las partes más lesionadas, entre ellas extremidades del cuerpo como las manos, pies y cabeza. ${ }^{25}$

De acuerdo con estudios que observaron la prevalencia de dolor e incomodidad en trabajadores en relación al frio del ambiente de trabajo, las quejas son más predominantes en las manos debido al contacto con los productos congelados. ${ }^{26-29}$

Los síntomas como estrés, malestar, somnolencia y rigidez de las manos reducen la destreza y sensibilidad de los dedos, aumentando gradualmente y predisponiendo a los trabajadores a condiciones de riesgos accidentales y problemas en la salud. ${ }^{30-32}$

La norma ISO 11079 menciona los valores límites para la temperatura superficial de la piel, así como las consecuencias al hacer caso omiso, influyendo en la salud del trabajador. ${ }^{33}$ La termografía mide las temperaturas superficiales de la piel desde 1987 por la Asociación Médica Americana. ${ }^{34}$ El método está compuesto por radiación infrarroja emitida por la piel y proporciona el análisis de la temperatura de la piel de forma no invasiva. ${ }^{34}$

\section{MATERIAL Y MÉTODOS}

Este estudio se basó en evaluaciones termográficas en seis partes corporales, localizados en los miembros superiores (derecho e izquierdo) de 19 funcionarios de frigoríficos en el estado de Santa Catarina (Brasil).

Las evaluaciones de las funcionarias quedaron concentradas en el primer turno, visto que las condiciones ambientales, o manipulación del producto y de los equipos presentan condiciones similares al del segundo turno, una vez que las condiciones de producción son fisca- lizadas diariamente por los funcionarios internos del Sistema de Inspección Federal, SIF. ${ }^{35}$

Para la realización del registro de las imágenes fue dispuesta una sala con temperatura ambiente con un valor de $23,2{ }^{\circ} \mathrm{C}$. Para monitorear la temperatura de la sala fue utilizado un medidor de estrés térmico de marca Instrutherm, modelo TGD-300.

Para evaluación de las temperaturas superficiales en los sitios corporales fue utilizado un medidor termográfico marca FLIR, modelo E40, temperaturas de -20 a $650^{\circ} \mathrm{C}$, con parámetros de calibración correspondientes a 0,95 de emisión, $20{ }^{\circ} \mathrm{C}$ de temperatura reflejada, $80 \%$ de humedad relativa. El equipo fue instalado en un triple, marca Instrutherm de color negro, a $85 \mathrm{~cm}$ del piso y $100 \mathrm{~cm}$ de distancia de una placa de acrílico utilizada como protección para las manos. La placa utilizada posee dimensiones de $30 \mathrm{~cm}$ de ancho por $25 \mathrm{~cm}$ de altura. Cada trabajadora participante fue retirada de la línea de producción y enviada para la sala de evaluación. Al llegar a la sala el procedimiento consistía en retirar los guantes, elevar las mangas de la blusa y colocar las manos en el frente de la placa de acrílico para registrar las fotos conforme a lo ilustrado en la Figura 1.

Figura 1.

Posición de los equipos y del participante en el instrumento de recolección de imágenes.

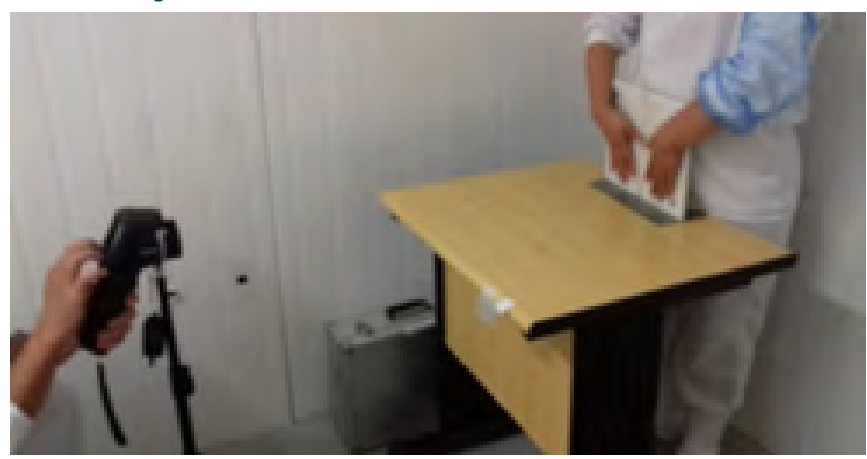

Posición de las participantes del estudio para la toma de foto de los segmentos.

Los datos fueron tratados con el programa del equipo y los resultados fueron analizados conforme a la Figura 2, que representa los puntos 1, 2 y 3 de cada mano donde fueron registradas las temperaturas superficiales de la piel.

Figura 2.

Imágenes de los sitios corporales analizados en el estudio.

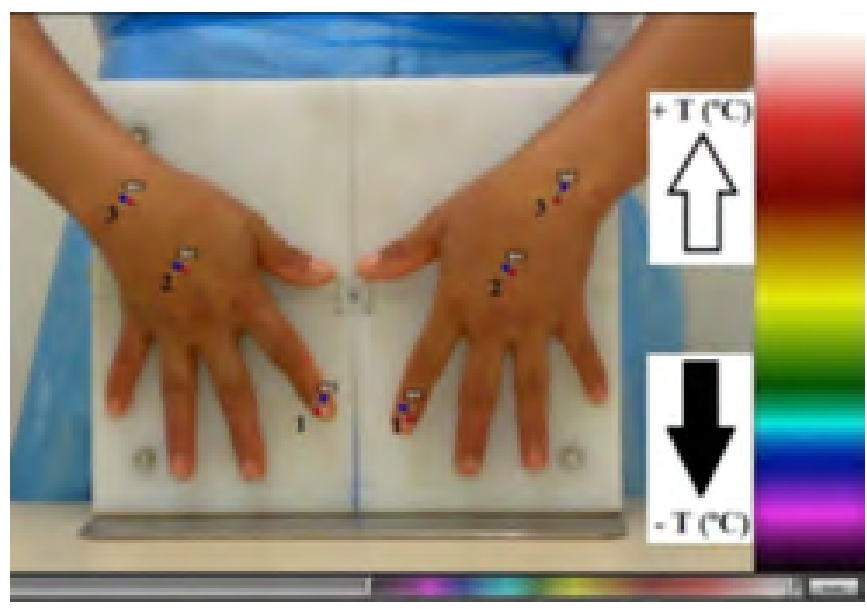

Sitios corporales analizados en el estudio: 1 dedos, 2 mano, 3 Muñeca. 
Fueron registradas las temperaturas de la primera falange distal del segundo dedo de cada mano, en el centro de ellas y en la región próxima a las muñecas, siendo todas localizadas en el lado dorsal de ambas manos. Para análisis de los datos, fue utilizada la temperatura promedio de cada sitio corporal separando la mano que porta el cuchillo de la mano que asegura el producto a ser cortado.

Los resultados de las temperaturas mínimas, máximas y promedio de cada punto, son representados por colores: el tono negro representa un color con menor temperatura $\mathrm{y}$, a medida que la temperatura se eleva, los tonos de colores también varían.

Para el análisis de los datos fue utilizada la estadística descriptiva y cálculo del valor $-\mathrm{P}, \alpha=0,05$ en análisis de varianza (ANOVA).

Para identificar posibles alteraciones patológicas en los sitios corporales que fueron analizados en las imágenes, se aplicó el cuestionario nórdico del dolor, donde los trabajadores participantes del estudio señalaban la parte con más sintomas de dolor. Para el análisis de los resultados fueron tabulados apenas los datos referentes a las muñecas, manos y dedos. Para análisis de los resultados fueron utilizados parámetros provenientes de una adaptación de las escalas de temperatura da ISO 1107933, basada en el estudio conducido por Lehmuskallio, Hassi e Kettunen. ${ }^{36}$ Para la medición de la temperatura superficial del mango del cuchillo y del producto manipulado por cada trabajador fue utilizado un termómetro digital infrarrojo marca Minipa modelo MT-350. Para medir la velocidad del aire en las salas fue utilizado un termohigrómetro marca instrutherm, modelo THAR-200.

Todas las funcionarias participantes aceptaron el término de consentimiento informado establecido con el protocolo de la investigación, presentado y aprobado por la comisión de Ética en investigación de la Universidad Federal de Santa Catarina.

\section{RESULTADOS}

Los resultados de la evaluaciones en los tres momentos de la recolección de datos fueron: Temperatura promedio medida del ambiente de trabajo de $11,2{ }^{\circ} \mathrm{C}$, velocidad promedio del aire de $0,26 / \mathrm{s}$, humedad relativa promedio de $80 \%$ temperatura promedio del producto manipulado de $4,8{ }^{\circ} \mathrm{C}$ y la temperatura promedio en el mango del cuchillo de $9,2{ }^{\circ} \mathrm{C}$.

Los resultados de las evaluaciones de las manos de una de las trabajadoras participantes de este estudio, conforme Figura 3. La imagen

\section{Figura 3.}

Imagen termográfica de los sitios corporales evaluados (dedo, centro de la mano y muñeca).

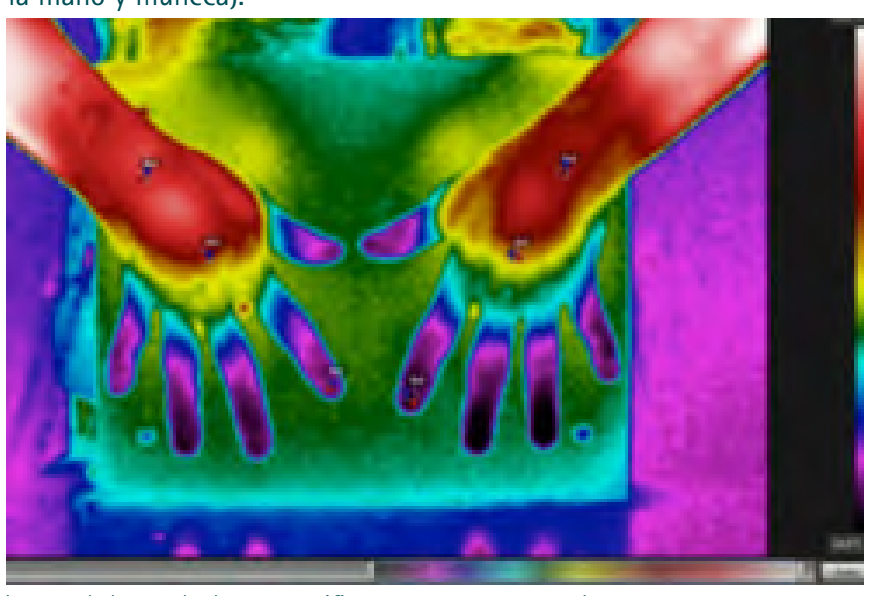

Imagen de los resultados termográficos y temperatura por colores. demuestra que no hay diferencias significativas de tonos de colores entre las manos.

Por medio del análisis de los resultados, de acuerdo con la indicación de los colores, es posible afirmar que la región de los dedos concibió el sitio corporal con las menores temperaturas, representado por el color púrpura o violeta con leve tono de negro. $\mathrm{Al}$ analizar las temperaturas en el centro de las manos y muñecas, representadas por el color rojo, es posible afirmar que estas presentan temperaturas mayores cuando son comparadas con las de los dedos.

Los resultados de los promedios y las variaciones de las temperaturas encontradas en los sitios corporales (izquierdo y derecho) son presentados en la Tabla 1.

Tabla 1.

Resultados del análisis estadístico de los promedios de las temperaturas por sitio corporal.

\begin{tabular}{|c|c|c|c|c|c|c|}
\hline $\begin{array}{l}\text { Sitio } \\
\text { corporal }\end{array}$ & $\begin{array}{l}\text { Lado del } \\
\text { miembro } \\
\text { superior } \\
\text { evaluado }\end{array}$ & Evaluaciones & $\begin{array}{c}\text { Número } \\
\text { de } \\
\text { muestras }\end{array}$ & $\begin{array}{c}\text { Promedio } \\
\text { de las } \\
\text { temperaturas } \\
\left({ }^{\circ} \mathrm{C}\right)\end{array}$ & Varianza & Valor - P \\
\hline \multirow[t]{2}{*}{ Dedo } & $\begin{array}{l}\text { Asegura } \\
\text { el cuchillo }\end{array}$ & $\begin{array}{c}\text { Primera } \\
\text { Segunda } \\
\text { Tercera }\end{array}$ & $\begin{array}{l}19 \\
19 \\
19\end{array}$ & $\begin{array}{l}19,37 \\
19,00 \\
18,78\end{array}$ & $\begin{array}{l}31,47 \\
25,81 \\
17,97\end{array}$ & 0,22 \\
\hline & $\begin{array}{l}\text { Asegura } \\
\text { el producto }\end{array}$ & $\begin{array}{c}\text { Primera } \\
\text { Segunda } \\
\text { Tercera }\end{array}$ & $\begin{array}{l}19 \\
19 \\
19\end{array}$ & $\begin{array}{l}19,07 \\
17,28 \\
16,67\end{array}$ & $\begin{array}{c}21,09 \\
14,82 \\
5,69\end{array}$ & 0,13 \\
\hline \multirow[t]{2}{*}{ Mano } & $\begin{array}{l}\text { Asegura } \\
\text { el cuchillo }\end{array}$ & $\begin{array}{c}\text { Primera } \\
\text { Segunda } \\
\text { Tercera }\end{array}$ & $\begin{array}{l}19 \\
19 \\
19\end{array}$ & $\begin{array}{l}28,12 \\
27,68 \\
27,30\end{array}$ & $\begin{array}{l}6,11 \\
5,39 \\
4,52\end{array}$ & 0,55 \\
\hline & $\begin{array}{l}\text { Asegura } \\
\text { el producto }\end{array}$ & $\begin{array}{c}\text { Primera } \\
\text { Segunda } \\
\text { Tercera }\end{array}$ & $\begin{array}{l}19 \\
19 \\
19\end{array}$ & $\begin{array}{l}28,49 \\
27,37 \\
27,12\end{array}$ & $\begin{array}{l}6,68 \\
6,89 \\
5,75\end{array}$ & 0,21 \\
\hline \multirow[t]{2}{*}{ Muñeca } & $\begin{array}{l}\text { Asegura } \\
\text { el cuchillo }\end{array}$ & $\begin{array}{c}\text { Primera } \\
\text { Segunda } \\
\text { Tercera }\end{array}$ & $\begin{array}{l}19 \\
19 \\
19\end{array}$ & $\begin{array}{l}29,66 \\
29,37 \\
29,18\end{array}$ & $\begin{array}{l}4,49 \\
3,12 \\
2,15\end{array}$ & 0,71 \\
\hline & $\begin{array}{l}\text { Asegura } \\
\text { el producto }\end{array}$ & $\begin{array}{c}\text { Primera } \\
\text { Segunda } \\
\text { Tercera }\end{array}$ & $\begin{array}{l}19 \\
19 \\
19\end{array}$ & $\begin{array}{l}29,41 \\
28,98 \\
28,54\end{array}$ & $\begin{array}{l}4,80 \\
5,27 \\
4,16\end{array}$ & 0,47 \\
\hline
\end{tabular}

Valores de los segmentos corporales que cumplen funciones (cuchillo y producto).

De acuerdo con los resultados, es posible observar en todas las evaluaciones que el valor -P calculado es mayor que el nivel de significancia de $\alpha=0,05$ escogido. En ese caso, no fueron encontradas evidencias sobre la influencia de algún factor en el valor de los promedios de las temperaturas encontradas, demostrando, por ejemplo, que los dedos de la mano de las 19 trabajadoras que utilizan el cuchillo presentan temperaturas promedio aproximadas en las evaluaciones realizadas durante el estudio. Con relación a las manifestaciones de dolor durante el estudio, los resultados están ilustrados en el Gráfico 1.

\section{Gráfico 1.}

Resultados de las quejas de dolor por parte del cuerpo conforme evaluación cuantitativa.

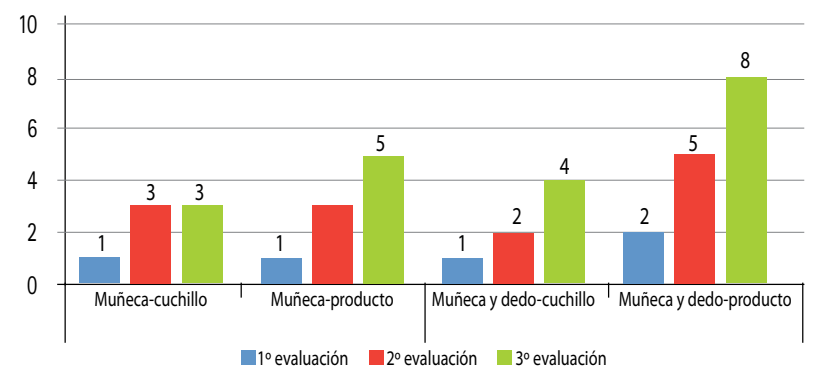

Clasificación y comparación de los dolores manifestados por los participantes del estudio. 
Se verifica en el Gráfico 1 que el mayor número de quejas de dolor ocurrió en la $3^{\circ}$ evaluación realizada en todos los sitios corporales analizados con la termografía, siendo que entre los tres evaluados (dedo, mano y muñeca) aquel que obtuvo el mayor número de quejas se concentró en las manos y dedos que aseguran el producto, con un total de 15 casos.

\section{DISCUSIÓN}

De acuerdo con los colores representados en la Figura 3, se verifica que en ambas manos los dedos poseen las menores temperaturas, representadas por el color púrpura con leve tono de negro, y en el centro de las manos y muñecas el color rojo. Se percibe que a pesar de ser la temperatura del producto usado inferior a la temperatura del mango del cuchillo, esa diferencia acabó no impactando en los resultados en los colores de temperatura entre las manos, en los tres sitios corporales evaluados. Probablemente ese efecto es sustituido por la diferencia de guantes utilizados en las manos izquierda y derecha o debido a la estabilización de temperaturas que ocurren en el ambiente laboral. Los resultados del análisis estadístico de los promedios de las temperaturas por sitio corporal evaluado apuntaron que los menores promedios de temperaturas se concentraron en los dedos de ambas manos; esto puede ser observado en el espectro de colores. Al analizar los resultados presentados en la temperatura promedio de los dedos, considerando ambos lados evaluados (derecho e izquierdo), se verifica que la variación de temperatura está comprendida entre 19,37-16,67 ${ }^{\circ} \mathrm{C}$; o sea, comprendidas en el intervalo considerado por el modelo adaptado de la ISO 1107933, con posibilidad de que existan trabajadores con sensación de incomodidad y dolor: Tabla 2.

Tabla 2.

Temperaturas promedio y análisis de las sensaciones de acuerdo con la adaptación de sensación térmica de la ISO 11079.

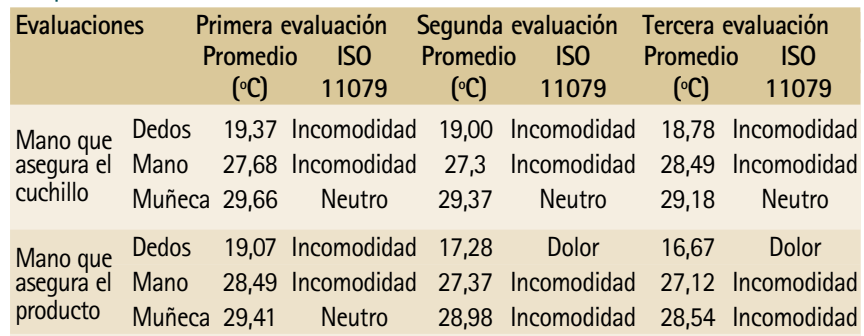

Valores comparados según la norma ISO 11079, con los segmentos que realizan las acciones y tipo de manifestación (incomodidad, dolor, neutro = incomodidad sin dolor o dolor sin incomodidad).

Se resalta que la ISO 1107933 recomienda que las temperaturas superficiales de los dedos deben estar encima de $24{ }^{\circ} \mathrm{C}$ para conservación de la función manual satisfactoria. Para completar este concepto, Holmer ${ }^{37}$ afirma que las temperaturas de los dedos bajo las condiciones de conformidad pueden ser aceptables, pero implicarán en perjuicio a la fuerza, destreza y coordinación, así como el aumento de la incidencia de quejas relacionadas a la sensación de dolor. Condición esta que probablemente justifica las sensaciones subjetivas de dolores de algunos trabajadores que se quejaron de las temperaturas promedio en la escala, señalando la sensación de incomodidad.

Comparando los resultados cuantitativos de la Tabla 2 con la tabulación de los resultados cualitativos representados en el Gráfico 1, se percibe que los dedos y mano que aseguran el producto se encuentran en un promedio de $4,8{ }^{\circ} \mathrm{C}$ en la parte más alcanzada por el frío y también se refiere al sitio corporal de queja más intensa de las trabajadoras. Además, es posible observar en la tercera evaluación realizada que las quejas de dolores aumentan con el transcurrir del tiempo de exposición, además de presentar los menores promedios de temperatura para ambas evaluaciones (derecha e izquierda) de los dedos y centro de las manos, lo que demuestra que también son las mayores quejas de dolores en este periodo.

Se nota en los resultados representados en la Tabla 2 y Gráfico 1 que no fue posible representar, en su totalidad, los datos subjetivos que pueden ser confirmados por los datos cuantitativos, lo que lleva a concluir que para algunos trabajadores la condición de incomodidad causada por el frio puede ser percibida como dolor. Debido a eso, cabe resaltar que cada persona tiene una capacidad fisiológica y metabólica, donde algunas pueden presentar mayor resistencia al frío que otras. Esta misma condición fue confirmada en el estudio realizado por Ramos. ${ }^{38}$ Esa investigación demostró que no siempre las percepciones subjetivas de temperatura del ambiente de trabajo, unidas a las condiciones proporcionadas a los trabajadores, estarán de acuerdo con la condición encontrada de temperatura superficial de la piel de las partes alcanzadas por el frío proveniente de las condiciones de trabajo.

Otro punto de vista relevante, además de la incomodidad y dolor causado por la exposición sin control al frío, puede ser la generación de síntomas como estrés, incomodidad, adormecimiento, rigidez de las manos de los trabajadores, reducción de destrezas, hormigueo, reducción de la sensibilidad de los dedos y flexibilidad de las articulaciones $^{30-32}$ y predisposición de los trabajadores a enfermedades, tales como úlceras, congelamiento, síndrome de Raynaud, urticaria por el frío, hongos dermatológicos, hipotermia, enfermedades respiratorias, entre otros. Un estudio que presenta una condicion similar de exposicion de los trabajadores a los riesgos de salud debido a la exposición al frío, y que puede ser citado como ejemplo, se refiere al de Kaminski M, Bourgine M, Zins M, Touranchet $\mathrm{A}^{39}$, donde fueron recolectados datos de 1474 trabajadores franceses de 17 plantas de sacrificios de aves y 6 fábricas de conservas desde 1987 hasta 1988, cuyos resultados demostraron que es posible en una población de hombres y mujeres expuestos al frío, sin vibración, detectar factores de riesgo para el síndrome de Raynaud, lo que lleva a concluir que pueden ser implementadas acciones que contribuyan a mantener las temperaturas dentro de los límites de tolerancia necesarios para que los trabajadores desarrollen las actividades con comodidad térmica, restando de esta forma acciones de mejoría a ser implementadas en los guantes de protección y en las pausas psico-fisiológicas.

Fanger $^{40}$ refuerza ese concepto de mejoras que deben ser implementadas al relatar en su estudio que para alcanzar la comodidad térmica es necesaria la combinación del nivel de actividad determinado por la naturaleza de trabajo con el aislamiento térmico de las vestimentas.

Teniendo en cuenta los datos de la empresa, su localización está en la zona climática mesotérmica en el mapa oficial "Brasil Climas" del IBGE, siendo así en virtud de la temperatura del local, promedio de $11,2{ }^{\circ} \mathrm{C}$; el ambiente de trabajo de la actividad analizada no es considerada por el decreto $\mathrm{N}^{\circ} 21$, del 26 de diciembre de 1994, del Ministerio de Trabajo y Empleo. ${ }^{41}$

Como alternativa de reducción de riesgo se encuentra el control del aislamiento térmico de la ropa industrial. Similar a ese estudio, citamos el realizado por Nielsen: ${ }^{42}$ los resultados también demostraron que la exposición de los dedos de los trabajadores a ambientes fríos es condición propicia a incomodidad y dolor. Sugirió que los estudios en las actividades evaluadas en esa investigación se orientan 
al uso de guantes gruesos, guantes con más aislamiento en la parte de los dedos, guantes con pequeños puntos de material aislante en la palma de la mano para disminuir las áreas de contacto con el objeto frío $\mathrm{y}$, consecuentemente, mejorar la adherencia en el agarre del producto disminuyendo la necesidad de fuerza.

Los resultados de esta investigación apuntan a la necesidad de efectuar el análisis cuantitativo de las condiciones en que los trabajadores expuestos a bajas temperaturas se encuentran en el ambiente de trabajo, en especial, en las actividades con exposición al frío. De acuerdo a los valores de los datos cuantitativos, es posible crear una relación con sensaciones subjetivas y evaluar la condición en la cual los trabajadores pueden estar expuestos en el desarrollo de sus actividades. También es posible establecer acciones para el control de la salud del trabajador, que pueden ser acciones administrativas o individuales, como, por ejemplo, la adecuación del tiempo de exposición al frio o equipos de protección individual adecuados a los riesgos.
Finalizando, concluimos que ese modelo de actividad, en las condiciones presentadas en ese estudio, exponen a los trabajadores a condiciones que pueden causar incomodidad, dolor, accidentes y posibles enfermedades relacionadas con la exposición al frío y que se vuelven preocupantes cuando se analizan las estadísticas oficiales de salud del trabajador y las estadísticas de expansión creciente de estas empresas tanto en demanda y producción como en número de funcionarios contratados.

\section{Agradecimientos}

A todos los trabajadores del Servicio de Seguridad y Medicina del Trabajo que contribuyeron para la realización de este proyecto, a la empresa que cedió el espacio para realizar la investigación, a los trabajadores participantes del estudio y a los profesores del Departamento de Ingeniería de Producción de la Universidad Federal de Santa Catarina, UFSC (Brasil).
1. Ministério da Agricultura Pecuária e Abastecimento (MAPA). Panorama da Avicultura Nacional e Perspectivas para o Setor [on line]. Brasilia: MAPA; 2017 [Acessado 18 jan 2017]. Disponivel em: http://www.agricultura.gov.br/assuntos/ sanidade-animal-e-vegetal/saude-animal/arquivos-das-publicacoesde-saude-animal/2-dr_-ariel-panorama-da-avicultura-nacional-e-perspectivas-para-o-setor.pdf/view

2. Instituto Brasileiro de Geografia e Estatística. Base de dados agregados (SIDRA) Pesquisa Trimestral do Abate de Animais; 2016 [on line]. Brasilia: IBGE; 2016 [Acessado 16 jan 2016]. Disponivel em: http://www.sidra.ibge.gov.br/bda/ tabela/listabl.asp?c=1093\&z=t\&0=24.

3. União Brasileira de Avicultura (UBABEF). Exportações brasileiras de carne de frango têm volume recorde para março, diz associação [on line]. São Paulo: ABPA; 2012 [Acessado 10 jan 2015]. Disponivel em: http://abpa-br.com.br/ setores/avicultura

4. Dejours $C$, Abdoucheli $E$, Jayet C. Psicodinâmica do trabalho. São Paulo: Atlas; 1994.

5. Bonde JP, Mikkelsen S, Andersen JH, Fallentin N, Baelum J, Svendsen SW, et al. Prognosis of shoulder tendonitis in repetitive work: a follow up study in a cohort of Danish industrial and service workers. Occup Environ Med 2003; 60:E8.

6. Caso MA, Ravaioli M, Veneri Lo. Esposizione a sovraccarico biomeccanico degli arti superiori: la valutazione del rischio lavorativo nei macelli avicoli. Prevenzione Oggi. 2007; 3(4):9-21.
7. Jakobi HR, Barbosa-Branco A, Bueno LF, Ferreira RGM, Camargo LMA. Beneficios auxilio-doença concedidos aos trabalhadores empregados no ramo de carne e pescado no Brasil em 2008. Cad. Saúde Pública. 2015; 31(1):194-207.

8. Armstrong J, Buckle P, Fine L, Hagberg BJ, Kilbom A, Kuorinka IA, et al. A conceptual model for workrelated neck and upper-limb musculoskeletal disorders. Scand J Work Environ Health. 1993; 19(2):73-84.

9. Bao $S$, Silverstein $B$, Cohen $M$. An electromyography study in three high risk poultry processing jobs. Int J Ind Ergonom. 2001; 27(6):375-385

10. Busnello GF, Dewes M. Doenças Osteomusculares Relacionadas à Atividade de Trabalhadores de Frigorificos de Frangos. Braz J Surg Clin Res. 2013; 4(3):27-32.

11. Frost $P$, Andersen $J H$, Nielsen VK. Occurrence of carpal tunnel syndrome among slaughterhouse workers. Scand J Work Environ Health 1998; 24(4):285-292.

12. Heck FM. Uma Geografia da Degradação do Trabalho: 0 Adoecimento dos Trabalhadores em Frigorificos. Percurso (Maringá). 2013; 5(1):3-31.

13. Juul-Kristernsen $B$, Fallentin $N$, Hansson GA. Physical workload during manual and mechanical deboning of poultry. Int J Ind Ergonom. 2002; 29(2):107-115.

14. Musolin K, Ramsey JG, Wassell JT, HARD DL. Prevalence of carpal tunnel syndrome among employees at a poultry processing plant. Appl ergon. 2014; 45(6):1377-1383.

15. Sarda SE, Ruiz RS, Kirtschig G. A Tutela Jurídica da Saúde dos Empregados de Frigoríficos: Considerações dos Serviços Públicos. Acta Fisiatr. 2009; 16(2):59-65. 


\section{REFERENCES}

16. Sommerich CM, Mcglothlin JD, Marras WS. Occupational risk factors associated with soft tissue disorders of the shoulder: a review of recent investigations in the literature. Ergonomics. 1993; 36(6):697-717.

17. Sundstrup $E_{1}$ Jakobsen $M D$, Andersen $C_{1}$, Jay $K$, Persson $R$, Aagaard $P_{1}$ Andersen LL. Participatory ergonomic intervention versus strength training on chronic pain and work disability in slaughterhouse workers: study protocol for a single-blind, randomized controlled trial. BMC musculoskel dis. 2013; 14(1):67-79.

18. Sundstrup $E_{1}$ Jakobsen MD, Jay K, Brandt M, Andersen LL. High Intensity Physical Exercise and Pain in the Neck and Upper Limb among Slaughterhouse Workers: Cross-Sectional Study. Biomed res int. 2014; 1:1-6.

19. Ministério da Previdência Social. Anuário Estatístico de Acidentes de Trabalho - AEAT Infologo: base de dados históricos de acidentes de trabalho [on line]. Brasilia: Ministerio da Previdência Social; 2015. [Acessado 12 jan 2016]. Disponivel em: http://www3.dataprev.gov.br/aeat/

20. Gallois NSP. Análise das condições de stress e conforto térmico sob baixas temperaturas em indústrias frigoríficas de Santa Catarina [dissertação]. Florianópolis: Universidade Federal de Santa Catarina; 2002. 125 p.

21. Reis PF. 0 trabalho repetitivo em frigorifico: utilização da estesiometria da mão como proposta para avaliação dos niveis de LER/DORT nas síndromes compressivas dos membros superiores. 2012 [dissertação]. Florianópolis: Universidade Federal de Santa Catarina; 2012. $137 \mathrm{p}$.

22. Sardá SE, Ruiz RS, Kirtschig G. A Tutela Jurídica da Saúde dos Empregados de Frigorificos: Considerações dos Serviços Públicos. Acta Fisiatr. 2009; 16(2):59-65.

23. IImarinen RE, Tammela EK. Design of functional work clothing for meat-cutters. Appl Ergon. 1990; 21(1):2-6.

24. Brasil. Ministério da Agricultura e do Abastecimento. Portaria № 210 de 10 Nov 1998. Dispõe sobre o regulamento técnico da inspeção tecnológica e higiênicosanitária de carnes de aves. Diário Oficial da União. Brasília (26 nov, 1998).

25. Launay JC, Savourey G. Cold adaptations. Ind health. 2009; 47(3):221-227.

26. Buzanello MR; Moro ARP. Slaughterhouse workers exposed to cold: proposal of reference thermography values for hands. Work. 2012; 41:2876-2881.

27. Buzanello MR. Influência de variáveis ambientais em frigorifico da unidade climática Subtropical Sul sobre os trabalhadores expostos a baixas temperaturas [dissertação]. Florianópolis: Universidade Federal de Santa Catarina; 2003. $132 \mathrm{p}$.
28. Holmér I. Extremity cooling and performance. Work in Cold Environments; Investigation Repport 31. Solna, Sweden: National Institute of Occupational Health, 1994. p. 49-55.

29. Holmér I. Cold stress: Part II - The scientific basis (knowledge base) for the guide. Int J Ind Ergon. 1994; 14:151-159.

30. Araujo AFDV, Zannoni C, Lima DBS, Santos EA, Dias ICL, Rodrigues ZMR. Identificação de fatores de riscos ocupacionais no processo de abate de bovinos. Cad Pesqui. 2013; 19(3):79-89.

31. lida I. Ergonomia: projeto e produção. 2a ed. São Paulo: Edgard Blucher; 2005.

32. Hassi J, Silkkila K, Ruokonen A, Leppäluoto J. The pituitary-thyroid axis in healthy men living under subarctic climatological conditions. J Endocrinol. 2001; 169(1):195-203.

33. ISO 11079. Ergonomics of the thermal environment - Determination and interpretation of cold stress when using required clothing insulation (IREO) and local cooling effects. Geneva: International Standards Organisation; 2007.

34. Hildebrandt $C$, Raschner $C$, Ammer K. An overview of recent application of medical infrared thermography in sports medicine in Austria. Sensors. 2010; 10(5):4700-4715.

35. NR36-Segurança e Saúde no Trabalho em Empresas de Abate e Processamento de Carnes e Derivados. Manuais de legislação Atlas; Segurança e Medicina do Trabalho. 73 ed. São Paulo: Atlas; 2014.

36. Lehmuskallio $E_{1}$ Hassi J, Ketunen P. The skin in the cold. Int J Circumpol Heal. 2002; 61:277-286.

37. Holmér I. Risk assessment in cold environments. Barents Newsl. 1999; 1:77-79.

38. Ramos E, Reis DC, Tirloni AS, Moro ARP. Thermographic analysis of the hands of poultry slaughterhouse workers exposed to artificially cold environment. Procedia Manuf. 2015; 3:4252-4259.

39. Kaminski $M$, Bourgine $M$, Zins $M$, Touranchet $A$, Verger $C$. Risk factors for raynaud's phenomenon among workers in poutry slaughterhouses and canning factories. Int J Epidemiol. 1997; 26(2): 371-380.

40. Fanger P. 0. Thermal comfort: analysis and applications in environmental engineering. New York: McGraw-Hill Book Company; 1970.

41. Brasil. Ministério do Trabalho e Emprego. Secretária de Segurança e Saúde no Trabalho, Portaria No. 21 de 26 Dec, 1994. Define o mapa oficial do Ministério do Trabalho para atender o disposto no art. 253 da CLT. (26 nov, 1994).

42. Nielsen R. Clothing and Thermal Environments. Field studies on industrial work in cool conditions. Appl Ergon. 1986; 17(1):47-57. 